\title{
乌s \\ Nanomechanical detection of nuclear magnetic resonance using a silicon nanowire oscillator
}

\author{
John M. Nichol, ${ }^{1}$ Eric R. Hemesath, ${ }^{2}$ Lincoln J. Lauhon, ${ }^{2}$ and Raffi Budakian ${ }^{1, *}$ \\ ${ }^{1}$ Department of Physics, University of Illinois at Urbana-Champaign, Urbana, Illinois 61801, USA \\ ${ }^{2}$ Department of Materials Science and Engineering, Northwestern University, Evanston, Illinois 60208, USA \\ (Received 1 November 2011; revised manuscript received 4 January 2012; published 13 February 2012)
}

\begin{abstract}
The authors report the use of a radio frequency (rf) silicon nanowire mechanical oscillator as a low-temperature nuclear magnetic resonance force sensor to detect the statistical polarization of ${ }^{l} \mathrm{H}$ spins in polystyrene. To couple the ${ }^{1} \mathrm{H}$ spins to the nanowire oscillator, a magnetic resonance force detection protocol was developed that utilizes a nanoscale current-carrying wire to produce large time-dependent magnetic field gradients as well as the rf magnetic field. Under operating conditions, the nanowire experienced negligible surface-induced dissipation and exhibited an ultralow force noise near the thermal limit of the oscillator.
\end{abstract}

DOI: 10.1103/PhysRevB.85.054414

PACS number(s): 76.60.-k, 07.55.-w, 81.07.Oj, 81.07.Gf

\section{INTRODUCTION}

Magnetic resonance force microscopy (MRFM) was proposed as a means of magnetic resonance imaging with the eventual goal of achieving the sensitivity to image individual molecules with atomic spatial resolution. ${ }^{1}$ MRFM detects the displacement of an ultrasensitive cantilever in response to the force generated by nuclear or electron spins in the presence of a magnetic field gradient. Most recently, MRFM imaging with spatial resolution below $10 \mathrm{~nm}$ and sensitivity to fewer than 100 net nuclear spins was demonstrated, ${ }^{2}$ far exceeding the capabilities of inductively detected magnetic resonance. Extending the resolution and sensitivity to the single proton level will likely require better force sensors, higher magnetic field gradients, or both. "Bottom-up" devices such as nanowires, ${ }^{3,4}$ nanotubes, ${ }^{5,6}$ and graphene ${ }^{7}$ oscillators have been proposed as next-generation force and mass sensors because of their relative ease of fabrication and potential for ultralow mechanical dissipation. In particular, silicon nanowires (SiNWs) have been shown to possess room temperature force sensitivity ${ }^{3}$ far below those of microscale cantilevers and thus offer a promising route to push MRFM closer to achieving the goal of molecular imaging.

The purpose of the present work is to establish nanowire oscillators as ultrasensitive cantilevers for MRFM detection. In particular, the SiNWs we study have extremely low intrinsic mechanical dissipation, and importantly for MRFM, the SiNWs experience negligible increase in dissipation as close as $10 \mathrm{~nm}$ to a surface. Another purpose of this study is to present an MRFM spin detection protocol, which uses a nanoscale current-carrying wire to generate time-dependent magnetic field gradients. This protocol enables MRFM with rf mechanical oscillators and may open new avenues for nanoscale magnetic resonance imaging.

\section{APPARATUS}

A schematic of the force detection apparatus is shown in Fig. 1. The SiNW used in this work was grown epitaxially on a $\mathrm{Si}[111]$ substrate using a controlled-diameter vapor-liquidsolid approach with silane as a precursor at $600{ }^{\circ} \mathrm{C} .{ }^{8}$ The SiNW was approximately $15 \mu \mathrm{m}$ long with a tip diameter of $35 \mathrm{~nm}$ and a base diameter of $200 \mathrm{~nm}$. Figure 2(a) shows several SiNWs representative of the type used here. Before use, the SiNW was annealed at $400^{\circ} \mathrm{C}$ for $2 \mathrm{~h}$ in forming gas (5\% hydrogen and $95 \%$ argon) to clean its surface and increase its quality factor. A thin coating of polystyrene on the SiNW tip [Fig. 2(b)] served as the sample containing ${ }^{1} \mathrm{H}$ spins. To create the coating, a droplet of polystyrene (Pressure Chemical Co. PS80317) dissolved in diethyl-phthalate (Alfa Aesar A17529) was placed on the tip of a glass micropipette and carefully brought into contact with the SiNW under an optical microscope approximately 20 times to build up a coating. To prevent reduction of the oscillator's quality factor, care was taken to ensure that the SiNW penetrated no more than $2 \mu \mathrm{m}$ into the droplet. The fundamental flexural mode of the SiNW had a spring constant $\mathrm{k}=650 \mu \mathrm{N} / \mathrm{m}$, a resonance frequency $\omega_{0} / 2 \pi=786 \mathrm{kHz}$, and an intrinsic quality factor $Q_{0}=2.5 \times 10^{4}$ at $8 \mathrm{~K}$. Before the polystyrene coating, the SiNW had a resonance frequency of $1.06 \mathrm{MHz}$, and the spring constant and quality factor were the same to within the experimental uncertainty.

To detect the displacement of the SiNW, a free-space interferometer coupled to a polarization-maintaining optical fiber ${ }^{3}$ was used (Fig. 1). The wavelength $\lambda=2 \mu \mathrm{m}$ was chosen to minimize optical absorption by the SiNW. The SiNW substrate was mounted on a three-axis piezoelectric positioner for coarse alignment with respect to the fiber and lens, which were also mounted on a two-axis piezoelectric bimorph scanner for fine optical alignment. The lens (Lightpath 370631) was used to focus the light exiting the optical fiber to a $2.5-\mu \mathrm{m}$-diameter spot on the SiNW. The current-carrying wire was mounted on a three-axis piezoelectric positioner and scanner for coarse and fine positioning with respect to the SiNW. The entire assembly was cooled to $4.2 \mathrm{~K}$ in a high-vacuum chamber $\left(<10^{-6} \mathrm{mbar}\right)$. The temperature of the SiNW was approximately $8 \mathrm{~K}$ with an incident optical power of roughly $1 \mu \mathrm{W}$.

\section{SURFACE DISSIPATION}

To produce the large magnetic field gradients required for sensitive spin detection, the distance between the sample and the gradient source must be very small, typically less than $100 \mathrm{~nm}$. At such small tip-surface separations, the cantilever dissipation usually increases considerably due to noncontact friction that arises from surface-induced forces fluctuating near $\omega_{0} \cdot{ }^{9,10}$ In general, the total mechanical dissipation is $\Gamma=\Gamma_{0}+$ $\Gamma_{\mathrm{s}}$, where $\Gamma_{0}=k / \omega_{0} Q_{0}$ is the intrinsic cantilever dissipation, 
(a)

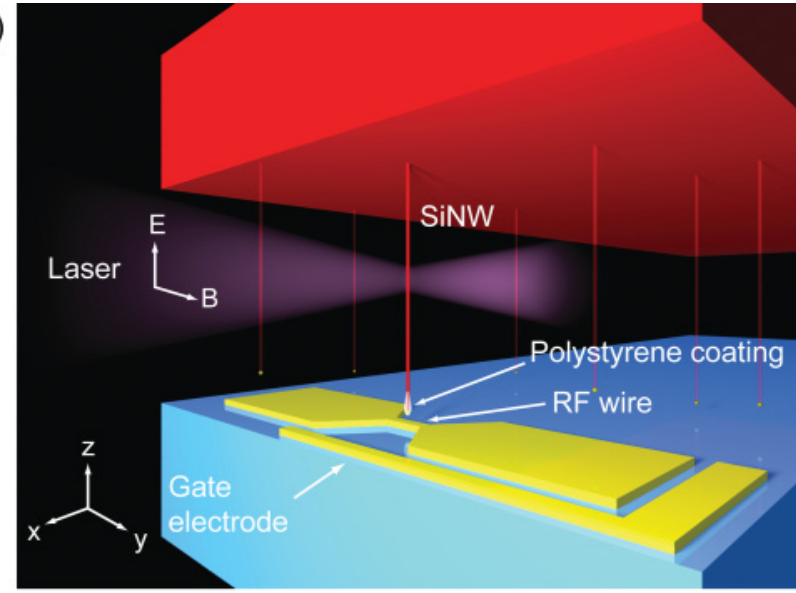

(b)

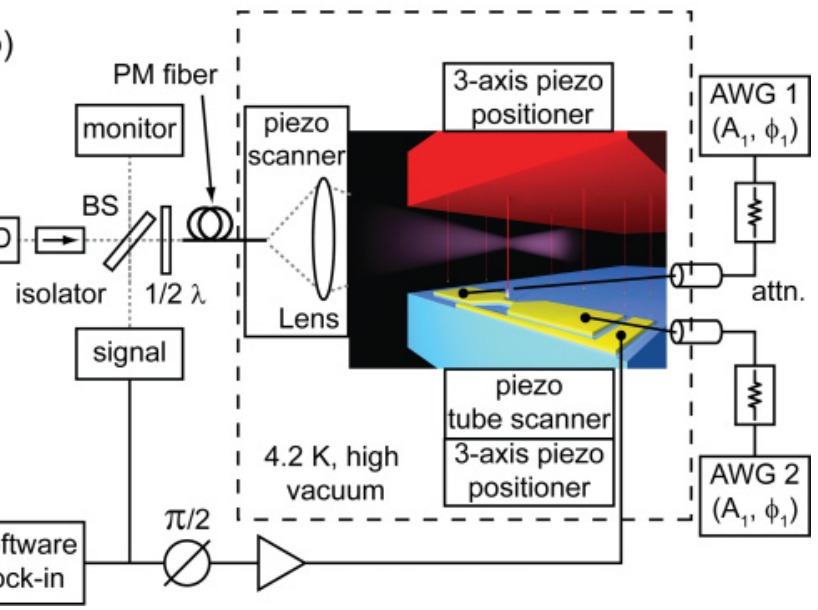

FIG. 1. (Color online) (a) Schematic of the experimental setup. Before the experiment, a single SiNW on the substrate was selected and coated with polystyrene. The SiNW tip was brought near the constriction in the rf wire. Focused, polarized laser light was used to detect the displacement of the specific SiNW with the polystyrene. (b) Experimental apparatus. A free-space interferometer coupled to an optical fiber was used to detect the displacement of the SiNW. In the interferometer, light from a $2-\mu \mathrm{m}$ laser diode (LD) passes through an optical isolator and a beam splitter (BS), couples into a polarization-maintaining (PM) optical fiber, and is focused with a lens onto the SiNW. Two arbitrary waveform generators (AWG 1 and AWG 2) with independently adjustable amplitudes and phases $\left(A_{1}\right.$, $\left.\phi_{1}, A_{2}, \phi_{2}\right)$ were used to drive the rf wire differentially.

and $\Gamma_{\mathrm{s}}$ is the dissipation caused by surface fluctuations. Near the surface, $\Gamma_{\mathrm{s}}$ can dominate the mechanical dissipation and can significantly increase the thermal force fluctuations $S_{\mathrm{F}}=4 k_{\mathrm{B}} T \Gamma$ experienced by the cantilever. Here, $k_{\mathrm{B}}$ is the Boltzmann constant and $T$ is the cantilever temperature. Because $S_{\mathrm{F}}$ determines the minimum force $\sqrt{S_{\mathrm{F}} \Delta f}$ detectable by the oscillator in a bandwidth $\Delta f$, noncontact friction presents a serious obstacle to improved sensitivity in MRFM.

To determine the effect of surface dissipation in SiNW oscillators, we measured the total dissipation of several SiNWs over polycrystalline gold surfaces at low temperature as a function of tip-surface separation [Fig. 3(a)]. The quality factor was determined by measuring the root mean square amplitude $x(t)$ of the thermal displacement, computing the autocorrelation of the oscillator energy $\left\langle x^{2}(t) x^{2}(t+\tau)\right\rangle$, and (a)

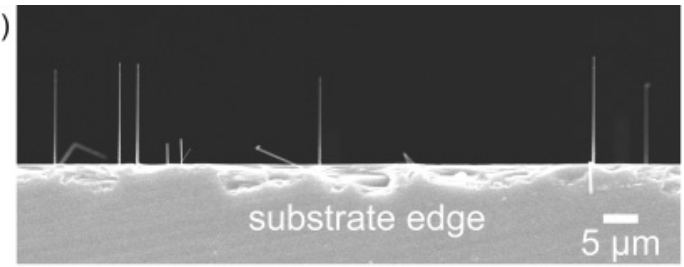

(b)

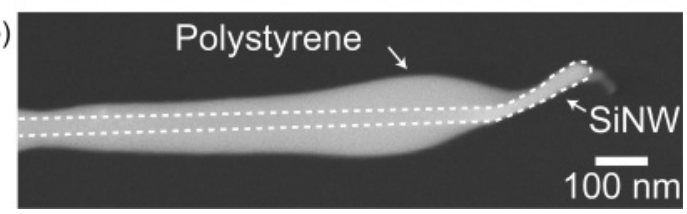

FIG. 2. (a) Scanning electron micrograph of a SiNW substrate showing several SiNWs representative of the type used in this study. (b) The tip of the SiNW used in this study with the polystyrene coating. The dashed lines indicate the outer diameter of the SiNW.

fitting to an exponential decay, ${ }^{9}$ where $\langle\cdots\rangle$ indicates a time average. Remarkably, SiNWs of the type we study maintain their ultralow native dissipation to within $10 \mathrm{~nm}$ from a surface. In fact, at a tip-surface spacing $d=7 \mathrm{~nm}$, a typical SiNW experiences nearly a factor of 80 less surface dissipation and factor of 250 less total dissipation than audio frequency cantilevers under similar conditions. ${ }^{9}$ The physical mechanisms underlying the reduced surface dissipation experienced by SiNWs are not completely clear. It may be that the small cross-sectional area of a SiNW decreases its coupling to the surface or that the spectral density of surface fluctuations is lower at $\mathrm{rf}$ frequencies than at audio frequencies.

Although SiNWs experience comparatively little surface dissipation, the frequency jitter of typical SiNWs increases to many times the thermal limit near a surface [Fig. 3(b)] as a result of $1 / \mathrm{f}$-like frequency fluctuations. ${ }^{11}$ Even in the presence of enhanced frequency jitter, however, the mean square thermal amplitude remains in agreement with the equipartition theorem $\left\langle x^{2}(t)\right\rangle=k_{\mathrm{B}} T / k$ to at least $d=15 \mathrm{~nm}$ [Fig. 3(b)]. Hence, the force fluctuations experienced by the SiNW obey the fluctuation-dissipation theorem, ${ }^{9}$ and the thermal fluctuations $S_{\mathrm{F}}$ remain the dominant source of force noise, even at small tip-surface separations.

\section{SPIN DETECTION PROTOCOL}

To take full advantage of the ultralow thermal force noise exhibited by the SiNWs, we have developed an MRFM spin detection protocol called MAGGIC (Modulated Alternating Gradients Generated with Currents). Using this scheme, we measure the $x$ component of the force on the SiNW from the longitudinal component of the spins: $F_{x}=\mu_{z} d B_{z} / d x$, where $d B_{z} / d x$ is the lateral magnetic field gradient and $\mu_{z}$ is the $z$ component of the spin magnetic moment. The MAGGIC protocol relies on AC currents passing through a constriction in a small wire, ${ }^{12}$ which we refer to as the rf wire, to generate both the rf field $B_{1}$ and a time-dependent gradient of the form $d B_{z}(t) / d x=G(t) \cos \left(\omega_{0} t\right)$ (Fig. 4), where $G(t)$ describes the amplitude modulation of the oscillating gradient. To minimize spurious excitation of the SiNW, $G(t)$ periodically reverses sign with a period $T_{\mathrm{AM}} \gg 2 \pi / \omega_{0}$ and ensures there is no 

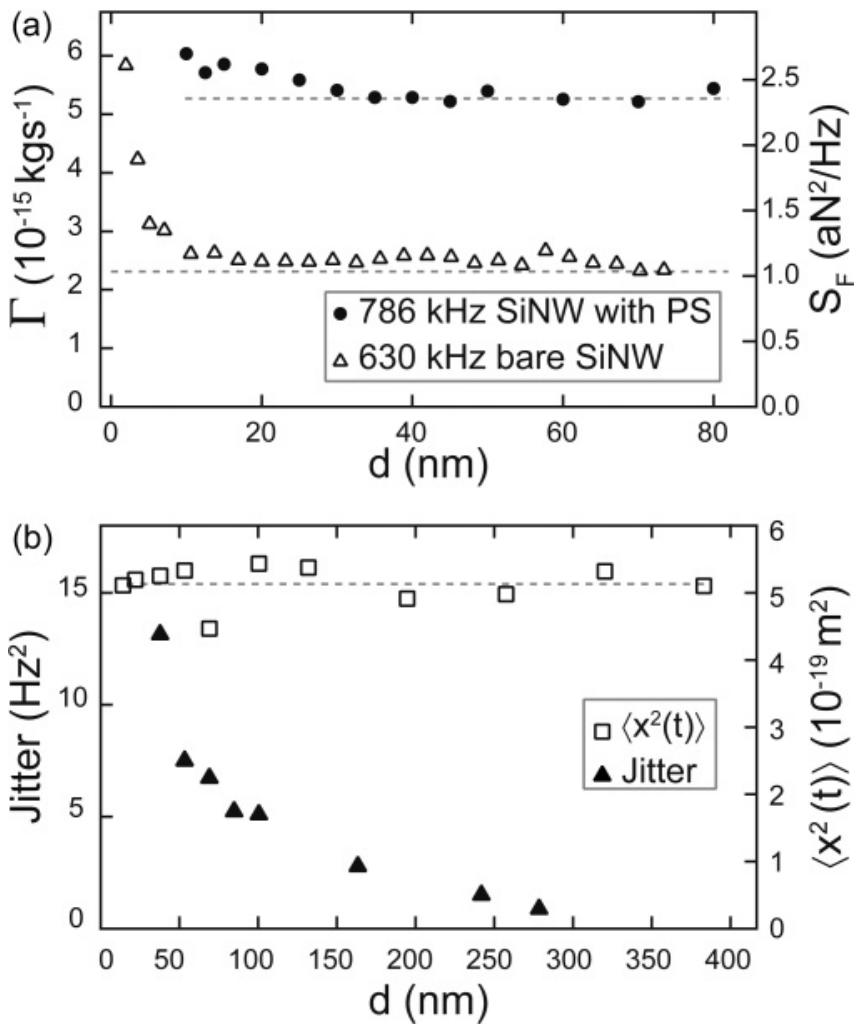

FIG. 3. (a) Total dissipation $\Gamma=k / \omega_{0} Q$ and thermal force noise $S_{\mathrm{F}}=4 k_{\mathrm{B}} T \Gamma$ for two different SiNWs: one with polystyrene (PS) $\left(\omega_{0} / 2 \pi=786 \mathrm{kHz}, k=650 \mu \mathrm{N} / \mathrm{m}\right)$ and one without $\left(\omega_{0} / 2 \pi=630 \mathrm{kHz}, k=215 \mu \mathrm{N} / \mathrm{m}\right)$. In each case, the surface was polycrystalline gold, and the $\mathrm{SiNW}$ temperature was $T=8 \mathrm{~K}$. Dashed lines indicate native dissipation as measured far away from a surface. Data are not shown for separations closer than $10 \mathrm{~nm}$ for the $786-\mathrm{kHz}$ SiNW because it seemed to bend slightly upon close approach, making an exact calibration of the distance difficult. (b) Mean square thermal displacement and integrated frequency fluctuations from 10 to $100 \mathrm{~Hz}$ for the $630-\mathrm{kHz} \mathrm{SiNW}$ at $T=8 \mathrm{~K}$. The contribution from thermal frequency fluctuations is $0.03 \mathrm{~Hz}^{2}$ in the integrated bandwidth. Although the frequency jitter increases near the surface, the mean square thermal amplitude remains constant and agrees with the equipartition theorem to at least $15 \mathrm{~nm}$ away from the surface (dashed line is the result from equipartition).

Fourier component of the voltage across the rf wire at $\omega_{0} \cdot{ }^{13}$ During the time $T_{\mathrm{OFF}}$ when $G(t)=0$, the spins on resonance are inverted adiabatically. Because the spins are reversed synchronously with $G(t)$, however, the force at $\omega_{0}$ does not change sign and resonantly drives the SiNW.

The time-dependent force exerted on the SiNW by a single spin is

$$
F(t)=\mu_{z}(t) G(t) \cos \left(\omega_{0} t\right),
$$

where $\mu_{z}(t)$ is the time-dependent spin $z$ component. $\mu_{z}(t)$ alternates in phase with $G(t)$ and also flips randomly with a correlation time $\tau_{m}$ due to statistical fluctuations. Hence, $\mu_{z}(t) G(t)=\mu h(t)|G(t)|$, where $\mu$ is the spin magnetic moment and $h(t)$ is a random telegraph function that takes on the values \pm 1 and has the following properties: $\langle h(t)\rangle=0$ and $\left\langle h^{2}(t)\right\rangle=1$. Because $G(t)$ varies slowly in time compared

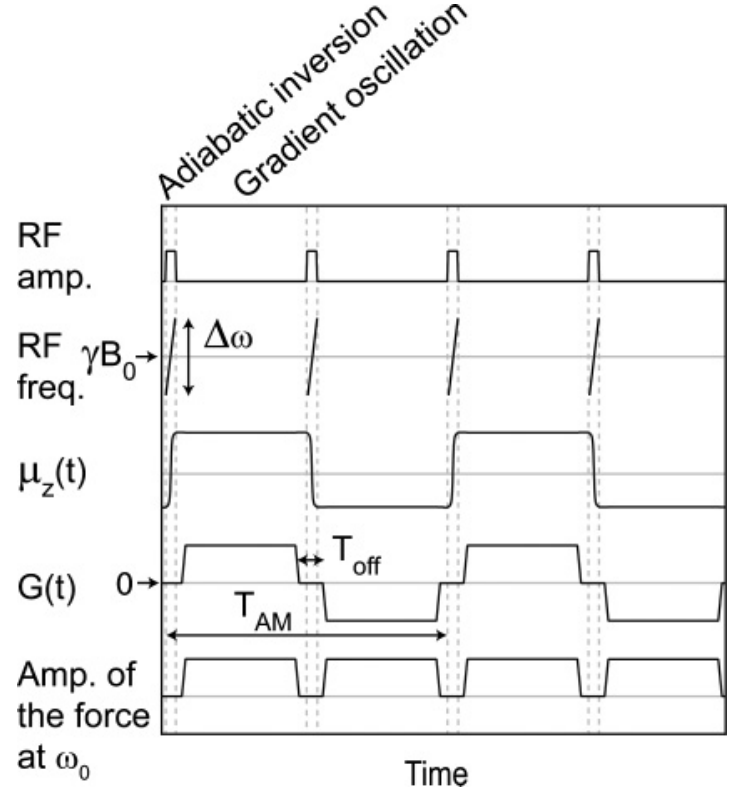

FIG. 4. MAGGIC timing diagram. Nuclear spins are adiabatically inverted by applying a frequency sweep through resonance using the rf wire. An AC current at $\omega_{0}$ generates the oscillating gradient. The gradient oscillation turns off for a duration $T_{\text {off }}$, the spins are inverted, and the gradient oscillation turns on again with the opposite sign. In the present experiment, the resonance frequency was $\gamma B_{0} / 2 \pi=$ $7.8 \mathrm{MHz}$. To generate the inversions, the rf frequency was swept through a bandwidth $\Delta \omega / 2 \pi=1.5-3 \mathrm{MHz}$ at a rate of $20-60$ $\mathrm{kHz} / \mu \mathrm{s}$. The gradient amplitude modulation frequency was typically $f_{\mathrm{AM}}=1 / T_{\mathrm{AM}}=300-800 \mathrm{~Hz}$, and the duty cycle was typically $D$ $\approx 0.8$.

with the SiNW oscillation and because, in practice, we choose $T_{\mathrm{AM}} \ll \tau_{m}$,

$$
\begin{aligned}
F(t) & =\mu D G_{\mathrm{pk}} h(t) \cos \left(\omega_{0} t\right)+\sum_{n=1}^{\infty} a_{ \pm n}(t) e^{i\left(\omega_{0} \pm 2 n \omega_{\mathrm{AM}}\right) t} \\
& =F_{0}(t) \cos \left(\omega_{0} t\right)+\text { sidebands. }
\end{aligned}
$$

Here, $G_{\mathrm{pk}}$ is the peak gradient and

$$
F_{0}(t) \equiv \mu D G_{\mathrm{pk}} h(t)
$$

is the amplitude of the force at $\omega_{0}$ averaged over the period $T_{\mathrm{AM}} / 2$. The quantity

$$
D \equiv \frac{2}{G_{\mathrm{pk}} T_{\mathrm{AM}}} \int_{0}^{T_{\mathrm{AM}} / 2}|G(t)| d t
$$

is the fractional amount of time the gradient is on and is approximately the duty cycle of the gradient modulation. Only the term $F_{0}(t) \cos \left(\omega_{0} t\right)$ in Eq. (2) resonantly excites the SiNW. Although the mean amplitude of this force vanishes, the mean square value $P$ is nonzero:

$$
P=\frac{\left\langle F_{0}^{2}(t)\right\rangle}{2}=\frac{\mu^{2} D^{2} G_{\mathrm{pk}}^{2}}{2} .
$$

The mean square displacement of the SiNW in response to this force is given by $\left\langle x^{2}(t)\right\rangle=P \frac{Q^{2}}{k^{2}}$.

A distinguishing feature of the MAGGIC protocol is the use of electric currents to generate strong, pulsed magnetic 
field gradients. This capability enables nuclear spin MRFM detection using rf oscillators, such as nanowires, without the need to modulate the nuclear magnetization at the cantilever frequency or oscillate the cantilever. Additionally, the absence of a static gradient during the adiabatic inversions means that the resonance condition is satisfied throughout the sample and that signal can be collected from all parts of the sample where the magnitude of the gradient is appreciable and $B_{1}$ is sufficient to invert the spins. The characteristic size of the detection region (several hundred nanometers in the present experiment) is related to the size of the constriction, which determines the spatial variation of the gradient and $B_{1}$.

Although we have not attempted imaging here, we note that a static gradient could be applied during the inversions to create a localized resonant slice, which could be scanned through the sample. Furthermore, the MAGGIC protocol is also compatible with well-established efficient magnetic resonance imaging schemes, such as Fourier ${ }^{14,15}$ and Hadamard ${ }^{16}$ encoding, which collect signal from many voxels simultaneously after a series of encoding pulses. In fact, MRFM using such schemes has previously been proposed ${ }^{17}$ and demonstrated ${ }^{18-20}$ with micrometer spatial resolution. The application of these techniques to nanometer-scale imaging will be the subject of future work.

To generate the strong time-dependent local fields and gradients for the MAGGIC protocol, we fabricated the rf wire by first sputtering a 5-nm-thick Ti/500-nm-thick Au film on a silicon substrate with a 500-nm-thick layer of thermal oxide. The contact pads and large wires were defined using argon ion milling with a photoresist etch mask. A focused ion beam was used to cut a 375-nm-wide and 500-nm-long constriction in the rf wire [Fig. 5(a)]. Before cutting with the focused ion beam, the device was annealed at $250^{\circ} \mathrm{C}$ for $3 \mathrm{~h}$ in dry nitrogen to decrease the resistivity of the film. The resistance of the device was approximately $2 \Omega$ at $4.2 \mathrm{~K}$.

The tip of the SiNW was positioned above the center of the constriction to maximize the magnitudes of both $B_{1}$ and $d B_{z} / d x$ [Fig. 5(b)]. The tip-surface separation was $80 \mathrm{~nm}$. The SiNW was electrostatically damped using a gate electrode [Figs. 1 and 5(a)] to $Q=1.3 \times 10^{4}$ to increase the detection bandwidth. ${ }^{21}$ Two arbitrary waveform generators (National Instruments PXI 5412) with independently adjustable amplitudes and phases were used to differentially drive the $\mathrm{rf}$ wire. Both generators had nominally the same amplitudes and opposite phases to ensure a voltage null at the constriction and minimize sideband excitation of the SiNW. (In the MAGGIC protocol, the voltage across the rf wire contains no Fourier component at $\omega_{0}$, but it does contain sidebands centered about $\omega_{0}$.) Fine adjustments were made to the amplitude and phase of each generator to further reduce the excitation of the SiNW. Approximately $67 \mathrm{~mA}$ of current, corresponding to a peak current density of $3.6 \times 10^{7} \mathrm{~A} / \mathrm{cm}^{2}$ through the constriction, generated both $B_{1}$ and the gradient oscillation. The current used here was limited by the compliance of the generators and not by the rf wire, which operated nearly continuously at this current density through the constriction for several weeks.

A small $(1-\mathrm{cm})$ superconducting solenoid inside the vacuum chamber provided the static field $B_{0}=0.183 \mathrm{~T}$ $\left(\gamma B_{0} / 2 \pi=7.8 \mathrm{MHz}\right)$ along the $z$ direction. In the MAGGIC protocol, the signal is maximized when $B_{0} \gg B_{x}$ and (a)

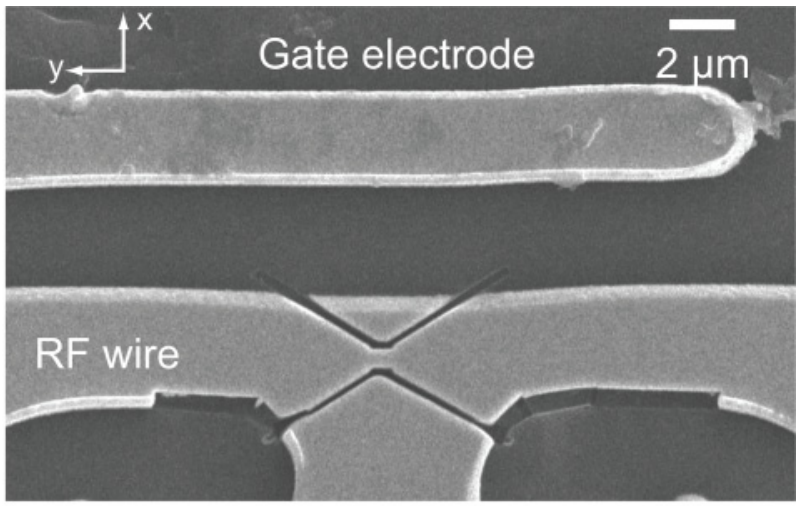

(b)

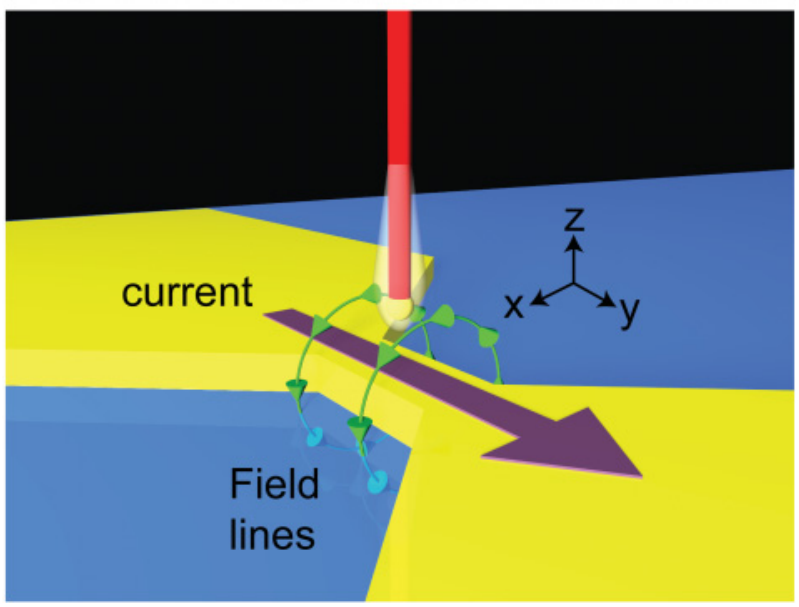

FIG. 5. (Color online) (a) Scanning electron micrograph of the rf wire, constriction, and gate electrode. The rf wire was fabricated from a 5-nm-thick Ti/500-nm-thick Au film on a silicon substrate with a 500-nm-thick layer of thermal oxide. A focused ion beam was used to create the constriction in the rf wire. Current passing through the constriction generates both the rf field and a time-varying field gradient to couple the spins to the SiNW. The gate electrode was used to electrostatically damp the SiNW. (b) During the experiment, the SiNW tip was positioned directly above the center of the constriction in the rf wire. At this location, both the magnitudes of $B_{1}$ and $d B_{z} / d x$ are maximized.

$\gamma B_{0} \gg \omega_{0}$, where $B_{x}$ is the amplitude of the field in the $x$ direction produced by the constriction during the gradient oscillation. As the gradient oscillates, the instantaneous total field cants by an angle $\alpha(t)=\tan ^{-1}\left(B_{x} \cos \left(\omega_{0} t\right) / B_{0}\right)$ away from the $z$ axis. Based on simulations of the Bloch equations, we find that the time dependence of $\mu_{z}$ in the absence of statistical fluctuations and adiabatic inversions can be approximated as $\mu_{z}(t) \approx \mu\left\{1-\left(\alpha_{\max } / 2\right)^{2}\left[1+\cos \left(2 \omega_{0} t\right)\right]\right\}$, provided $\alpha_{\max }=$ $\tan ^{-1}\left(B_{x} / B_{0}\right)<1$ and $\gamma B_{0} \gg \omega_{0}$. As a result of this time dependence, the amplitude of the force at $\omega_{0}$ in Eq. (3) is replaced by $F_{0}(t) \rightarrow F_{0}(t) R$, and peak spin signal power in Eq. (5) is replaced by $P \rightarrow P R^{2}$, where $R=1-3 \alpha_{\max }^{2} / 8$. In the present experiment, $B_{x}<0.035 \mathrm{~T}, \alpha_{\max }<11^{\circ}$, and $R>0.99$. Thus, the peak signal power was not significantly reduced.

\section{RESULTS}

The spin signal was measured by demodulating the displacement signal using a software-based lock-in amplifier referenced to the gradient oscillation. Shown in Fig. 6 is the 
force power spectrum from the in-phase lock-in channel. The MRFM signal from the statistically polarized ${ }^{1} \mathrm{H}$ spins in the polystyrene appears as a peak at $0 \mathrm{~Hz}$. The total spectrum $S=$ $S_{\text {spin }}+S_{\mathrm{N}}$ is the sum of the spin signal, which is well described by a Lorentzian function ${ }^{22} S_{\text {spin }}=4 \tau_{m} P /\left[1+\left(2 \pi \tau_{m} f\right)^{2}\right]$, and a constant noise background $S_{\mathrm{N}}$. By fitting the data, we find $P=5.9 \pm 1.2 \mathrm{aN}^{2}$, and the spin relaxation time $\tau_{m}=1.04 \pm 0.03 \mathrm{~s}-\mathrm{a}$ factor of two greater than what has previously been reported in polystyrene. ${ }^{23}$ The increased spin relaxation time may occur because there is no static field gradient during the spin manipulation, or because in the MAGGIC protocol, the spins spend relatively little time in the rotating frame.

The force noise in the detection quadrature was $S_{\mathrm{N}}=3.8 \pm$ $1.2 \mathrm{aN}^{2} / \mathrm{Hz}$, slightly above the thermal noise power, which was $S_{\mathrm{F}}=2.4 \pm 0.6 \mathrm{aN}^{2} / \mathrm{Hz}$ at $8 \mathrm{~K}$. The observed force noise is significantly lower than what has been measured in micron scale cantilevers ${ }^{2}$ operating at $300 \mathrm{mK}$. The uncertainty estimates are based on the uncertainties in the measurements of the quality factor, temperature, and spring constant of the SiNW. In subsequent experiments, we confirmed that the excess noise above $S_{\mathrm{F}}$ was caused by phase noise of the arbitrary waveform generators exciting the SiNW. We have since implemented the MAGGIC protocol using a single waveform generator with a $0-180^{\circ}$ rf splitter to differentially drive the constriction. With the splitter, the voltage phase noise produced by the generator cancels itself at the constriction, and the excess noise is substantially reduced.

To measure the magnitude of $B_{1}$, we applied nutation pulses $^{12}$ (Fig. 7) to observe Rabi oscillations (Fig. 8). From the period of the Rabi oscillations, we find that the average $B_{1}=8.8 \mathrm{mT}$ (rotating frame). As expected, $\tau_{m}$ increased with increasing $B_{1}$ (Fig. 6 upper inset). As a check, we simulated the magnitude of the signal based on the geometry of the constriction and polystyrene coating. We modeled the constriction as an infinitely long wire with a $500 \mathrm{~nm} \times$ $375 \mathrm{~nm}$ rectangular cross section. Finite element analysis using

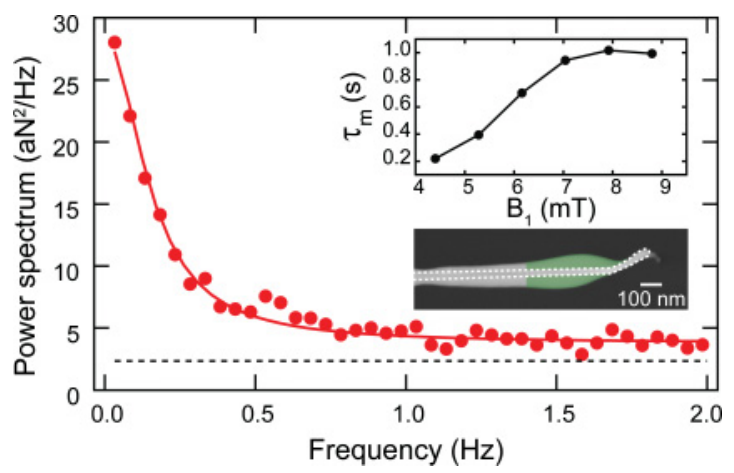

FIG. 6. (Color online) Spectrum from the in-phase lock-in channel showing the statistically polarized spin signal and fit to a Lorentzian at $T=8 \mathrm{~K}, B_{0}=0.183 \mathrm{~T}$, and $\gamma B_{0} / 2 \pi=7.8 \mathrm{MHz}$. The dashed line is the calculated thermal force noise. The displacement noise from the interferometer contributed approximately $1.0 \mathrm{aN}^{2} / \mathrm{Hz}$ to the force noise. This contribution has been subtracted to display only the force noise of the oscillator. Upper inset: dependence of the spin relaxation time on the rf amplitude. Lower inset: The shaded region of the polystyrene contributes $90 \%$ of the observed signal power.

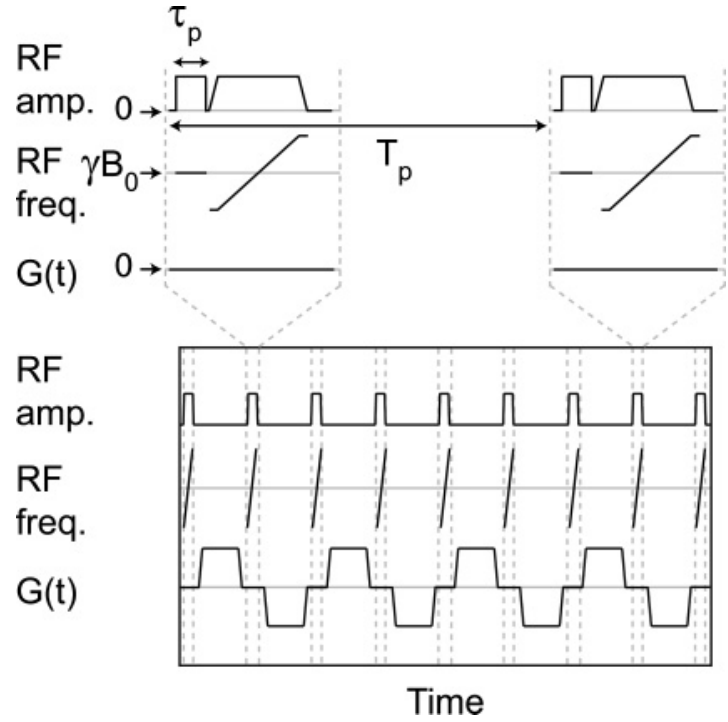

FIG. 7. Nutation pulse sequence. Rf pulses at $\omega_{\mathrm{rf}}=\gamma B_{0}$ of variable length $\tau_{\mathrm{p}}$ were inserted in the MAGGIC protocol every $T_{\mathrm{p}}=$ $500 \mathrm{~ms}$, or every 175 modulation periods at $f_{\mathrm{AM}}=350 \mathrm{~Hz}$. The period $T_{\mathrm{p}}$ was chosen to be less than the spin lock lifetime $\tau_{m}$.

COMSOL Multiphysics (COMSOL, Inc.) of the actual wire geometry, including the adjacent metal islands, has confirmed that the infinite wire model reproduces the relevant fields and gradients to within $10 \%$ in the region of space occupied by the polystyrene sample. The infinite wire model was used for ease of computation. The shape of the polystyrene coating was extracted from scanning electron micrographs, and the signal power was computed as

$$
P=\frac{\rho \mu^{2} D^{2} \cos ^{2}(\theta)}{2} \sum_{\mathbf{r} \in V} \Delta V\left(d B_{z}(\mathbf{r}) / d x\right)^{2} .
$$

Here, $\rho=4.9 \times 10^{28} \mathrm{~m}^{-3}$ is the ${ }^{1} \mathrm{H}$ density in polystyrene, $\mu$ $=1.4 \times 10^{-26} \mathrm{~J} / \mathrm{T}$ is the proton magnetic moment, $D$ is given by Eq. (4), $\Delta V$ is the volume element of the simulation, and $\theta$ is the tilt angle of the SiNW away from the $z$ axis (approximately $15^{\circ}$, as measured with scanning electron microscopy). To

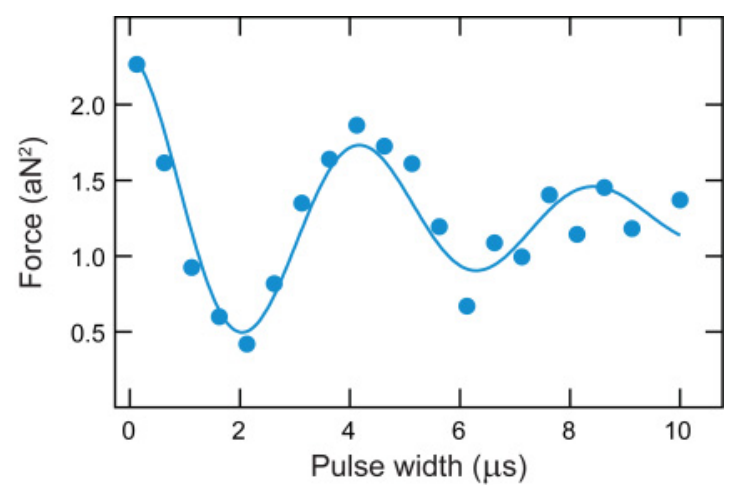

FIG. 8. (Color online) Rabi oscillations for an rf current of $42 \mathrm{~mA}$. To determine the period of the Rabi oscillations, we fit the data to an exponentially decaying cosine. From the measured oscillation period of $4.25 \mu \mathrm{s}$, we determine that $B_{1}=5.5 \mathrm{mT}$. Thus, for $67 \mathrm{~mA}$ through the construction, $B_{1}=8.8 \mathrm{mT}$. 
calculate the signal power, we summed over all coordinates in the sample volume $V$.

The calculated signal power is $11 \mathrm{aN}^{2}$. The agreement with experiment is reasonable given the absence of free parameters in the calculation. The discrepancy may be due to imperfect adiabatic inversions or improper positioning of the SiNW tip over the constriction. Were the SiNW tip actually $200 \mathrm{~nm}$ away in the $x$ direction from the center of the constriction, for example, the calculated signal power would be $7 \mathrm{aN}^{2}$. Although we have not directly measured the magnetic field gradient, our calculations indicate that $d B_{z} / d x=1.2 \times$ $10^{5} \mathrm{~T} / \mathrm{m}$ at a distance of $80 \mathrm{~nm}$. Because the gradient falls off rapidly away from the constriction, the polystyrene closest to the SiNW tip contributes most of the signal power (Fig. 6, lower inset). In the future, stronger field gradients in excess of $10^{6} \mathrm{~T} / \mathrm{m}$ should be possible with smaller constrictions supporting current densities in the $10^{9} \mathrm{~A} / \mathrm{cm}^{2}$ range; such large current densities have been reported in nanoscale metal constrictions whose size is small compared with the electron mean free path. ${ }^{24}$

\section{CONCLUSION}

We have demonstrated a new route to ultrasensitive MRFM detection using SiNW rf oscillators and the MAGGIC spin detection protocol. The use of bottom-up nanomechanical oscillators as force detectors opens the door for greatly improved force sensitivity. Furthermore, the ability to generate large time-dependent field gradients may enable efficient methods for nanoscale magnetic resonance imaging. Together, these new tools promise to advance MRFM closer toward the goal of molecular imaging.

\section{ACKNOWLEDGMENTS}

The authors thank Tyler Naibert for fabrication assistance and John Mamin and Dan Rugar for helpful discussions. This work was supported by the Department of Physics at the University of Illinois and the Frederick Seitz Materials Research Laboratory. Work at Northwestern University was supported by the National Science Foundation Grant No. DMI0507053 through the NIRT program. *budakian@illinois.edu

${ }^{1}$ J. A. Sidles, J. L. Garbini, K. J. Bruland, D. Rugar, O. Zuger, S. Hoen, and C. S. Yannoni, Rev. Mod. Phys. 67, 249 (1995).

${ }^{2}$ C. L. Degen, M. Poggio, H. J. Mamin, C. T. Rettner, and D. Rugar, Proc. Natl. Acad. Sci. USA 106, 1313 (2009).

${ }^{3}$ J. M. Nichol, E. R. Hemesath, L. J. Lauhon, and R. Budakian, Appl. Phys. Lett. 93, 193110 (2008).

${ }^{4}$ E. Gil-Santos, D. Ramos, J. Martinez, M. Fernandez-Regulez, R. Garcia, A. San Paulo, M. Calleja, and J. Tamayo, Nat. Nanotechnol. 5, 641 (2010).

${ }^{5}$ V. Sazonova, Y. Yaish, H. Ustunel, D. Roundy, T. A. Arias, and P. L. McEuen, Nature 431, 284 (2004).

${ }^{6}$ K. Jensen, K. Kim, and A. Zettl, Nat. Nanotechnol. 3, 533 (2008).

${ }^{7}$ J. S. Bunch, A. M. van der Zande, S. S. Verbridge, I. W. Frank, D. M. Tanenbaum, J. M. Parpia, H. G. Craighead, and P. L. McEuen, Science 315, 490 (2007).

${ }^{8}$ D. E. Perea, E. Wijaya, J. L. Lensch-Falk, E. R. Hemesath, and L. J. Lauhon, J. Solid State Chem. 181, 1642 (2008).

${ }^{9}$ B. C. Stipe, H. J. Mamin, T. D. Stowe, T. W. Kenny, and D. Rugar, Phys. Rev. Lett. 87, 096801 (2001).

${ }^{10}$ S. Kuehn, R. F. Loring, and J. A. Marohn, Phys. Rev. Lett. 96, 156103 (2006).

${ }^{11}$ S. M. Yazdanian, N. Hoepker, S. Kuehn, R. F. Loring, and J. A. Marohn, Nano Lett. 9, 2273 (2009).
${ }^{12}$ M. Poggio, C. L. Degen, C. T. Rettner, H. J. Mamin, and D. Rugar, Appl. Phys. Lett. 90, 263111 (2007).

${ }^{13}$ Oscillating electric fields produced by the rf wire can strongly drive the SiNW due to the presence of uncompensated charge on the SiNW.

${ }^{14}$ A. Kumar, D. Welti, and R. R. Ernst, J. Magn. Reson. 18, 69 (1975).

${ }^{15}$ D. I. Hoult, J. Magn. Reson. 33, 183 (1979).

${ }^{16}$ L. Bolinger and J. S. Leigh, J. Magn. Reson. 80, 162 (1988).

${ }^{17}$ J. G. Kempf and J. A. Marohn, Phys. Rev. Lett. 90, 087601 (2003).

${ }^{18}$ K. W. Eberhardt, C. L. Degen, and B. H. Meier, Phys. Rev. B 76, 180405 (2007)

${ }^{19}$ K. W. Eberhardt, A. Hunkeler, U. Meier, J. Tharian, S. Mouaziz, G. Boero, J. Brugger, and B. H. Meier, Phys. Rev. B 78, 214401 (2008).

${ }^{20}$ R. Joss, I. T. Tomka, K. W. Eberhardt, J. D. van Beek, and B. H. Meier, Phys. Rev. B 84, 104435 (2011).

${ }^{21}$ J. L. Garbini, K. J. Bruland, W. M. Dougherty, and J. A. Sidles, J. Appl. Phys. 80, 1951 (1996).

${ }^{22}$ D. Rugar, R. Budakian, H. J. Mamin, and B. W. Chui, Nature 430, 329 (2004).

${ }^{23}$ F. Xue, P. Peddibhotla, M. Montinaro, D. P. Weber, and M. Poggio, Appl. Phys. Lett. 98, 163103 (2011).

${ }^{24}$ K. S. Ralls and R. A. Buhrman, Phys. Rev. Lett. 60, 2434 (1988). 